OPEN

SUBJECT AREAS: APPLIED MICROBIOLOGY DNA RECOMBINATION GENETIC ENGINEERING METABOLIC ENGINEERING

Received

10 June 2013

Accepted

16 December 2013

Published

15 January 2014

Correspondence and requests for materials should be addressed to

L.Q.H. (huangluqi@

263.net) or X.L.Z.

(zhang_xl@tib.cas.cn)

* These authors contributed equally to this work.

\section{Producing aglycons of ginsenosides in bakers' yeast}

\author{
Zhubo Dai 1,2*, Beibei Wang 1,2,3*, Yi Liv' ${ }^{1,2,3}$, Mingyu Shi ${ }^{1,2,3}$, Dong Wang ${ }^{1,2,3}$, Xianan Zhang ${ }^{4,5}$, Tao Liu' ${ }^{1,2}$, \\ Luqi Huang ${ }^{4} \&$ Xueli Zhang ${ }^{1,2}$
}

${ }^{1}$ Tianjin Institute of Industrial Biotechnology, Chinese Academy of Sciences, ${ }^{2}$ Key Laboratory of Systems Microbial Biotechnology, Chinese Academy of Sciences, ${ }^{3}$ College of Biotechnology, Tianjin University of Science and Technology, ${ }^{4}$ National Resource Center for Chinese Materia Medica, China Academy of Chinese Medical Sciences, ${ }^{5}$ School of Traditional Chinese Medicine, Capital Medical University.

Ginsenosides are the primary bioactive components of ginseng, which is a popular medicinal plant that exhibits diverse pharmacological activities. Protopanaxadiol, protopanaxatriol and oleanolic acid are three basic aglycons of ginsenosides. Producing aglycons of ginsenosides in Saccharomyces cerevisiae was realized in this work and provides an alternative route compared to traditional extraction methods. Synthetic pathways of these three aglycons were constructed in $S$. cerevisiae by introducing $\beta$-amyrin synthase, oleanolic acid synthase, dammarenediol-II synthase, protopanaxadiol synthase, protopanaxatriol synthase and NADPH-cytochrome P450 reductase from different plants. In addition, a truncated 3-hydroxy-3-methylglutaryl-CoA reductase, squalene synthase and 2,3-oxidosqualene synthase genes were overexpressed to increase the precursor supply for improving aglycon production. Strain GY-1 was obtained, which produced $17.2 \mathrm{mg} / \mathrm{L}$ protopanaxadiol, $15.9 \mathrm{mg} / \mathrm{L}$ protopanaxatriol and $21.4 \mathrm{mg} / \mathrm{L}$ oleanolic acid. The yeast strains engineered in this work can serve as the basis for creating an alternative way for producing ginsenosides in place of extractions from plant sources.

insenosides are the primary bioactive components of ginseng, which is a popular medicinal plant. Ginsenosides are a group of triterpenoids that exhibit diverse pharmacological effects on the central nervous, endocrine, cardiovascular and immune systems ${ }^{1,2}$. Dammarane-type tetracyclic ginsenosides (Rb1, Rb2, Rc, Rd, Re, Rf, Rg1, Rh2 and Rg3) are the major constituents, while Ro, which belongs to oleanane-type pentacyclic ginsenosides, is a minor component of Panax ginseng ${ }^{3}$. These Dammarane-type ginsenosides are divided into two groups according to their aglycon structure: protopanaxadiol (Rb1, Rb2, Rc, Rd, Rh2 and Rg3) and protopanaxatriol (Re, Rf, and Rg1) .

Currently, ginsenosides are mainly produced through their extraction from ginseng roots. Wild ginseng roots are scarce (i.e., they are endangered species in Asia and North America), but most commercial ginseng roots are collected from farms that cultivate ginseng in fields ${ }^{4}$. However, cultivating ginseng is time-consuming, labourintensive and is influenced by many conditions such as soil, climate, pathogens and pests ${ }^{4}$.

Bakers' yeast (Saccharomyces cerevisiae) has been commonly used as a leavening agent in baking bread and bakery products, where it converts fermentable sugars that are present in dough into carbon dioxide and ethanol. In addition, because $S$. cerevisiae has been genetically and physiologically characterised and multiple genetic engineering tools exist, this microorganism is an ideal host for the heterologous production of valuable natural products and can provide an alternative and attractive route compared to traditional extraction methods ${ }^{5-8}$. With the development of metabolic engineering and synthetic biology tools, many natural products have been successfully synthesised in S. cerevisiae c,9-12. $^{\text {. }}$

The first committed step in triterpenoid biosynthesis is the cyclisation of 2,3-oxidosqualene. This reaction is catalysed by specific oxidosqualene cyclases (OSCs), e.g., dammarenediol-II synthase (DDS), $\beta$-amyrin synthase (bAS), $\alpha$-amyrin synthase (aAS), lansterol synthase (LAS) and lupeol synthase (LUS) ${ }^{13-15}$. In P. ginseng, cyclisation of 2,3-oxidosqualene to dammarenediol-II by DDS is the first reaction towards the biosynthesis of dammarane-type ginsenosides ${ }^{16,17}$. Dammarenediol-II is further converted to protopanaxadiol by protopanaxadiol synthase (PPDS), which is a cytochrome P450 enzyme that catalyses the hydroxylation of dammarenediol-II at the C-12 position (Fig. 1) ${ }^{3}$. Protopanaxadiol is further converted to protopanaxatriol by protopanaxatriol synthase (PPTS) (Fig. 1) ${ }^{16}$. In contrast, cyclisation of 2,3-oxidosqualene to $\beta$-amyrin by $\beta$-amyrin synthase (bAS) is the first reaction towards the biosynthesis of oleanane-type ginsenosides. $\beta$-amyrin is further converted to oleanolic acid by oleanolic acid synthase (OAS, a P450 enzyme), which catalyses a three-step, sequential oxidation 


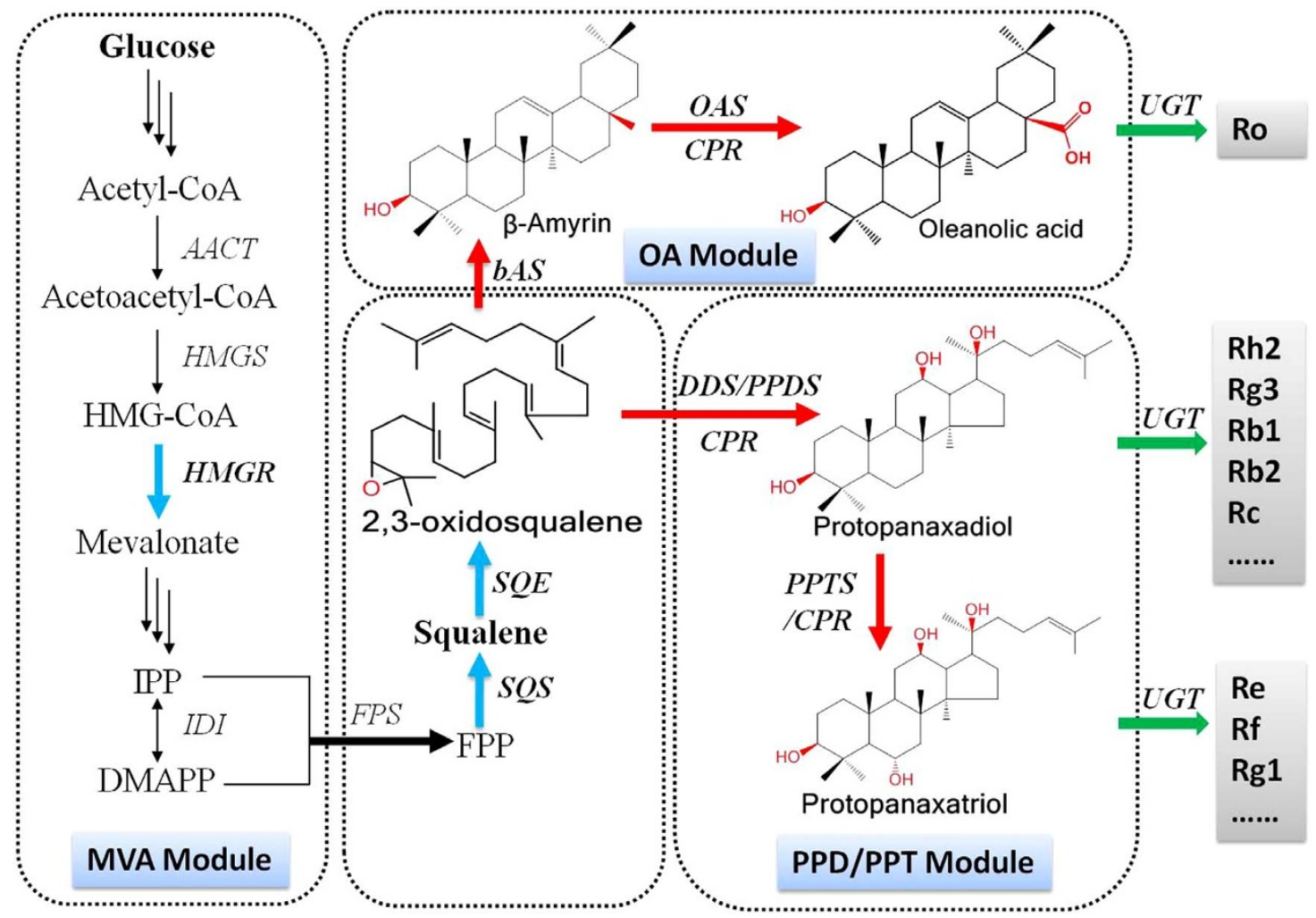

Figure 1 Biosynthetic pathways for protopanaxadiol, protopanaxatriol and oleanolic acid production in metabolically engineered S. cerevisiae. Single arrows represent one-step conversions, while triple arrows represent multiple steps. Bold, blue arrows represent over-expressed yeast endogenous genes. Bold, red arrows represent exogenous plant genes that were introduced into S. cerevisiae. OA module includes of G. glabra bAS, M. truncatula OAS and A. thaliana CPR genes, while PPD/PPT module includes A. thaliana CPR and DDS, PPDS and PPTS of P. ginseng genes. Intermediates: HMG-CoA, 3hydroxy-3-methylglutaryl coenzyme A; IPP, isopentenyl pyrophosphate; DMAPP, dimethylallyl pyrophosphate; FPP, farnesyl diphosphate; OA, oleanolic acid; PPD, protopanaxadiol; and PPT, protopanaxatriol. Enzymes: HMGR, 3-hydroxy-3-methylglutaryl-CoA reductase; SQS, squalene synthase; SQE, squalene epoxidase; bAS, $\beta$-amyrin synthase; OAS, oleanolic acid synthase; CPR, cytochrome P450 reductase; DDS, dammarenediol-II synthase; PPDS, protopanaxadiol synthase; and PPTS, protopanaxatriol synthase.

at the C-28 position of $\beta$-amyrin (Fig. 1) ${ }^{14,15}$. These aglycons are further converted to ginsenoside compounds by uridine diphosphate glycosyltransferases (UGTs) ${ }^{18,19}$.

In this work, $S$. cerevisiae was metabolically engineered for the efficient production of these three aglycons by introducing $\beta$-amyrin synthase, oleanolic acid synthase, dammarenediol-II synthase, protopanaxadiol synthase, protopanaxatriol synthase and NADPHcytochrome P450 reductase from different plants. Genes coding for OAS from Medicago truncatula and DDS, PPDS and PPTS from $P$. ginseng have been previously demonstrated to be functional in yeast ${ }^{3,16,17}$. In addition, precursor supplies were increased to improve aglycon production. The yeast strains engineered in this work can serve as the basis for creating an alternative way for producing ginsenosides in place of extraction from plant sources.

\section{Results}

Increasing IPP and DMAPP supplies for terpenoid production in S. cerevisiae. Triterpenoids are derived from two common building blocks, isopentenyl diphosphate (IPP) and dimethylallyl diphosphate (DMAPP), which are synthesised through the mevalonic acid (MVA) pathway in S. cerevisiae (Fig. 1). Most IPP and DMAPP precursors enter the ergosterol synthetic pathway ${ }^{20-22}$. When cultivated in YPD medium with $2 \%$ glucose for 7 days, strain BY4742-TRP produced $9.6 \mathrm{mg} / \mathrm{L}$ squalene, $2.1 \mathrm{mg} / \mathrm{L}$ lanosterol and $10.1 \mathrm{mg} / \mathrm{L}$ ergosterol (Fig. 2).

3-hydroxy-3-methylglutaryl-CoA (HMG-CoA) reductase is a key rate-limiting enzyme in the MVA pathway, and overexpression of a truncated HMG-CoA reductase gene ( $t H M G 1)$ has commonly been used to increase the carbon flux through the MVA pathway ${ }^{11,25}$. This strategy had been used to successfully increase the production of many terpenoids, including sesquiterpenoids $s^{7,11,26,27}$, diterpenoids ${ }^{9,10,28}$, triterpenoids $\mathrm{s}^{29,30}$ and carotenoids ${ }^{31}$.

The $t H M G 1$ gene (controlled by the PGK1 promoter) and the LYS2 gene (controlled by the TEF1 promoter) were integrated into the chromosome of BY4742-TRP at the $\delta D N A$ site to increase IPP and DMAPP supplies for better terpenoid production. The resulting strain, BY-T1 (Table 1), produced $150.9 \mathrm{mg} / \mathrm{L}$ squalene, $5.4 \mathrm{mg} / \mathrm{L}$ lanosterol and $42.0 \mathrm{mg} / \mathrm{L}$ ergosterol, which were 15.7-, 2.6- and 4.2fold higher than that of the parent strain (Fig. 2). This result demonstrated that overexpressing $t H M G 1$ significantly improved terpenoid production, and the engineered strain could be used for further production of aglycons of ginsenosides.

Construction of the $\beta$-amyrin synthetic pathway. $\beta$-amyrin serves as the basic precursor of oleanane-type triterpenoids ${ }^{18}$. $\beta$-amyrin is synthesised from 2,3-oxidosqualene in some medicinal plants by 2,3oxidosqualene cyclases (OSCs) ${ }^{13-15,23}$. S. cerevisiae can produce 2,3oxidosqualene from IPP and DMAPP through farnesyl diphosphate synthase (encoded by ERG20), squalene synthase (encoded by ERG9) and squalene epoxidase (encoded by ERG1) (Fig. 1).

Squalene synthase and squalene epoxidase are two key enzymes for triterpenoid synthesis. Squalene synthase is the first enzyme dedicated to synthesis of sterols in yeast, while squalene epoxidase catalyses the first oxygenation step in ergosterol biosynthesis and is suggested to represent one of the rate-limiting enzymes in this pathway $^{32}$. Overexpressing the squalene synthase gene had been used to enhance the production of $\beta$-amyrin in yeast ${ }^{29,30}$ and the production 


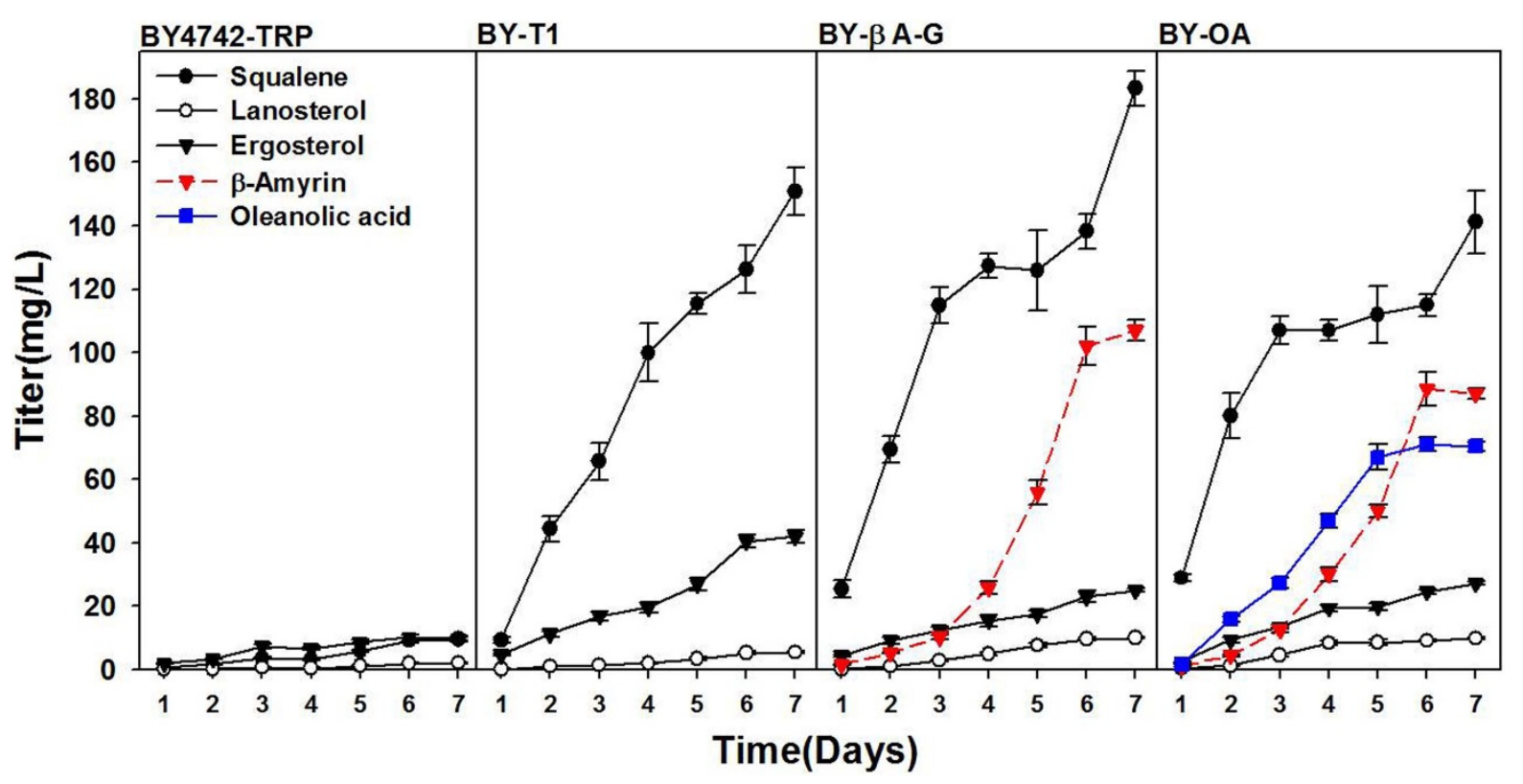

Figure $2 \mid$ Production of squalene, lanosterol, ergosterol, $\beta$-amyrin and oleanolic acid by engineered $S$. cerevisiae strains BY4742-TRP, BY-T1, BY- $\beta$ A-G and BY-OA. All strains were cultivated in YPD medium with $2 \%$ glucose for 7 days. Three replicates were performed, and the error bars represented standard deviation. All these five chemicals were measured in the four strains. $\beta$-amyrin and oleanolic acid were not detected in strains BY4742-TRP and BY-T1. Oleanolic acid was not detected in strain BY- $\beta A-G$.

of phytosterols and triterpenoids in plants ${ }^{33-35}$. Furthermore, overexpressing the squalene epoxidase gene had been used to increase sterol production ${ }^{21,25}$.

To construct the $\beta$-amyrin synthetic pathway and improve its production in $S$. cerevisiae, two different $\beta$-amyrin synthase genes of Glycyrrhiza glabra and P. ginseng, with the S. cerevisiae squalene synthase and squalene epoxidase genes, were integrated into the chromosome of strain BY-T1 at rDNA sites, resulting in strains BY- $\beta A-G$ and BY- $\beta A-P$ (Table 1 ), respectively. When cultivated in YPD medium with $2 \%$ glucose for 7 days, GC/MS analysis of cell extractions of both strains confirmed the production of $\beta$-amyrin (Fig. S1). Strain BY- $\beta$ A-P produced $1.9 \mathrm{mg} / \mathrm{L} \beta$-amyrin with a yield of $0.2 \mathrm{mg} / \mathrm{g}$ (data not shown), while strain BY- $\beta A-\mathrm{G}$ produced $107.0 \mathrm{mg} / \mathrm{L} \beta$-amyrin with a yield of $9.3 \mathrm{mg} / \mathrm{g}$ DCW (Fig. 2). Strain BY- $\beta A-G$ also produced $183.4 \mathrm{mg} / \mathrm{L}$ squalene, $10.0 \mathrm{mg} / \mathrm{L}$ lanosterol and $25.0 \mathrm{mg} / \mathrm{L}$ ergosterol (Fig. 2).

To verify that increasing 2,3-oxidosqualene supply did improve $\beta$ amyrin synthesis, the $\beta$-amyrin synthase gene of G. glabra (controlled by the PGK1 promoter) and the LYS 2 gene (controlled by the TEF1 promoter) were integrated into the chromosome of strain BY4742-TRP at $r D N A$ sites. The resulting strain, BY- $\beta A-C K$ (Table 1), produced $77.7 \mathrm{mg} / \mathrm{L} \beta$-amyrin, which was $73 \%$ of that produced by strain BY- $\beta$ A-G (Fig. S2). In addition, strain BY- $\beta$ ACK produced $34.2 \mathrm{mg} / \mathrm{L}$ squalene, $1.4 \mathrm{mg} / \mathrm{L}$ lanosterol and $12.1 \mathrm{mg} /$ L ergosterol, which were $19 \%, 14 \%$ and $48 \%$ of those produced by strain BY- $\beta A-G$ (Fig. S2).
Construction of the oleanolic acid synthetic pathway. To construct the oleanolic acid synthetic pathway in S. cerevisiae, the oleanolic acid synthase gene $(O A S)$ of Medicago truncatula ${ }^{13}$ (controlled by TEF1 promoter), with a cytochrome P450 reductase gene of Arabidopsis thaliana $(A t C P R 1)^{24}$ (controlled by $T D H 3$ promoter) and a new copy of the G. glabra $\beta$-amyrin synthase gene (controlled by $P G K 1$ promoter) were integrated into the chromosome of strain BY$\beta A-G$ at the Trp1 site, resulting in strain BY-OA (Table 2). After cultivation in YPD medium with $2 \%$ glucose for 7 days, LC/MS analysis of the cell extraction of strain BY-OA confirmed the production of oleanolic acid (Fig. S3). Strain BY-OA produced $71.0 \mathrm{mg} / \mathrm{L}$ oleanolic acid with a yield of $6.1 \mathrm{mg} / \mathrm{g}$ DCW and $88.6 \mathrm{mg} / \mathrm{L} \beta$-amyrin with a yield of $7.6 \mathrm{mg} / \mathrm{g}$ DCW (Fig. 2). This strain also produced $141.2 \mathrm{mg} / \mathrm{L}$ squalene, $9.8 \mathrm{mg} / \mathrm{L}$ lanosterol and $27.3 \mathrm{mg} / \mathrm{L}$ ergosterol (Fig. 2).

Construction of the synthetic pathways of all three aglycons. To construct the protopanaxadiol and protopanaxatriol synthetic pathways in strain BY-OA, the $P$. ginseng dammarenediol-II synthase gene (PgDDS, controlled by $P G K 1$ promoter), $P$. ginseng protopanaxadiol synthase gene ( $P g P P D S$, controlled by TEF1 promoter), $P$. ginseng protopanaxatriol synthase gene (PgPPTS, controlled by FBA1 promoter) and AtCPR1 (controlled by $T D H 3$ promoter) genes were integrated into the chromosome of strain BY-OA at the His3 site, resulting in strain GY-1 (Table 1). After cultivation in YPD medium with $2 \%$ glucose for 5 days, LC/MS analysis of the cell

Table 1 | Strains used in this study

Name

Description

Source

BY4742

BY4742-TRP

BY-T 1

BY- $\beta A-P$

BY- $\beta A-G$

BY- $\beta A-C K$

BY-OA

GY-1
$M A T \alpha$, his341, leu2 40 , lys2 40, MET15, ura3 40

Deletion of the Trpl gene of BY4742

BY4742-TRP, $\delta D N A:: P_{P G K 1}-t H M G 1-T_{A D H 1}-P_{T E F 1}-L Y S 2-T_{C Y C 1}$

BY-T1, rDNA:: $P_{P G K 1}-P g b A S-T_{A D H 1}-P_{T D H 3}-E R G 1-T_{T P I 1}-P_{T E F 1}-E R G 9-T_{C Y C 1}$

BY-T1, rDNA:: $P_{P G K 1}-G g b A S-T_{A D H 1}-P_{T D H 3}-E R G 1-T_{T P I 1}-P_{T E F 1}-E R G 9-T_{C Y C 1}$

BY4742-TRP, rDNA:: $P_{P G K 1}-G g b A S-T_{A D H 1}-P_{T E F 1}-L Y S 2-T_{C Y C 1}$

BY- $\beta A-G$, Trp $1:: P_{P G K 1}-G g b A S-T_{A D H 1}-P_{T D H 3}-A+C P R 1-T_{T P 1}-P_{T E F 1}-M+O A S-T_{C Y C 1}$

BY-OA, His3:: $P_{P G K 1}-P g D D S-T_{A D H 1}-P_{F B A 1}-S y n P g P P T S-T_{T D H 2}-P_{T D H 3}-A t C P R 1-T_{T P I 1}-P_{T E F 1}-S y n P P D S-T_{C Y C 1}$
Brachmann et al. ${ }^{38}$

This study

This study

This study

This study

This study

This study

This study 


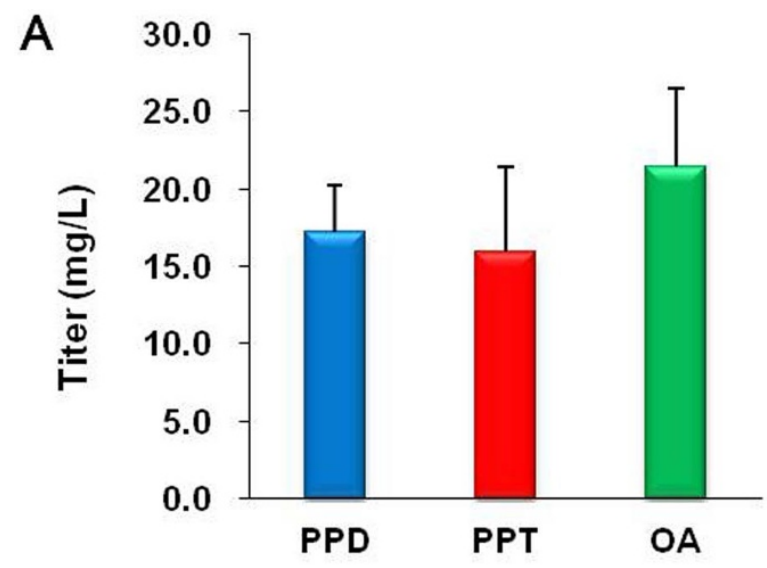

B

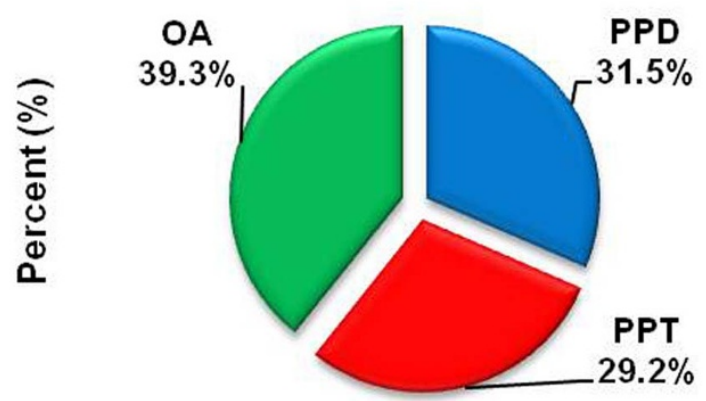

Figure 3 Production of protopanaxadiol, protopanaxatriol and oleanolic acid by engineered $S$. cerevisiae strain GY-1. (A) Titers obtained after fermentation for 7 days; (B) Proportion of protopanaxadiol, protopanaxatriol and oleanolic acid within the total aglycons. Three replicates were performed, and the error bars represented standard deviation.

extraction of strain GY-1 confirmed the production of protopanaxadiol and protopanaxatriol (Fig. S4 and S5). Strain GY-1 produced $17.2 \mathrm{mg} / \mathrm{L}$ protopanaxadiol with a yield of $1.4 \mathrm{mg} / \mathrm{g}$ DCW, and $15.9 \mathrm{mg} / \mathrm{L}$ protopanaxatriol with a yield of $1.3 \mathrm{mg} / \mathrm{g}$ DCW. The oleanolic acid titer decreased from 71.0 to $21.4 \mathrm{mg} / \mathrm{L}$, and its yield decreased from 6.1 to $1.8 \mathrm{mg} / \mathrm{g}$ DCW (Fig. 3A). The proportion of protopanaxadiol, protopanaxatriol and oleanolic acid within the total aglycons was $31.5 \%, 29.2 \%$ and $39.3 \%$, respectively (Fig. 3B). In addition, this strain produced $96.0 \mathrm{mg} / \mathrm{l}$ squalene, $11.1 \mathrm{mg} / \mathrm{l}$ lanosterol, $16.3 \mathrm{mg} / \mathrm{l}$ ergosterol, $24 \mathrm{mg} / \mathrm{l} \beta$-amyrin and $7.9 \mathrm{mg} / \mathrm{l}$ dammarenediol-II (data not shown).

\section{Discussion}

S. cerevisiae is an ideal host for the heterologous production of valuable natural products. To be economically comparable to the extraction from plant sources, heterologous production of ginsenosides in yeast with high titer and productivity is required. Because 2,3-oxidosqualene is a basic precursor for ginsenoside synthesis, its sufficient supply is very important. In this work, three commonly used engineering strategies for increasing the 2,3-oxidosqualene supply were performed to improve $\beta$-amyrin production. After overexpressing the $t H M G 1, E R G 9$ and $E R G 1$ genes, $\beta$-amyrin production increased $38 \%$ and all squalene-derived terpenoids increased $159 \%$ (Fig. S2), demonstrating that these three enzymes were essential for increasing the 2,3-oxidosqualene supply to improve triterpenoid production.

$\beta$-amyrin serves as the basic, oleanane-type triterpenoid precursor to variable downstream products ${ }^{18}$. Through the introduction of $\beta$-amyrin synthase of Artemisia annua, the overexpression of the tHMG1 gene and down-regulation of the lanosterol synthase gene (encoded by ERG7), an engineered S. cerevisiae was obtained that produced $6 \mathrm{mg} / \mathrm{L} \beta$-amyrin ${ }^{29}$. By introducing $\beta$-amyrin synthase of Pisum sativum and overexpressing the phosphomevalonate kinase (encoded by ERG8), squalene synthase (encoded by ERG9) and acetyl-CoA carboxylase (encoded by HFA1) genes, an engineered S. cerevisiae was obtained that produced $3.93 \mathrm{mg} / \mathrm{L} \beta$-amyrin ${ }^{30}$. To the best of our knowledge, strain BY- $\beta A-G$ had the highest $\beta$-amyrin titer that was achievable by microbial fermentation. It was suggested that the $\beta$-amyrin synthase of G. glabra was better at producing $\beta$ amyrin than $A$. annua, $P$. sativum and $P$. ginseng. This suggestion was reasonable because G. glabra contains a large amount of glycyrrhizin $(2-8 \% \text { of the dry weight })^{23,36}$, while the other three plants contain minimal levels of oleanane-type triterpenoids ${ }^{16}$.

For strain BY- $\beta A-G$, squalene was produced with high productivity $(1.60 \mathrm{mg} / \mathrm{L} \cdot \mathrm{h})$, while $\beta$-amyrin had a relatively lower productivity $(0.14 \mathrm{mg} / \mathrm{L} \cdot \mathrm{h})$ during the first 72 hours (Fig. 2). From 72 to $144 \mathrm{~h}$, squalene productivity decreased $80 \%$ to $0.32 \mathrm{mg} / \mathrm{L} \cdot \mathrm{h}$, while $\beta$-amyrin productivity increased 9.1 -fold to $1.27 \mathrm{mg} / \mathrm{L} \cdot \mathrm{h}$. One possibility was that $\beta$-amyrin synthase activity was relatively lower for the first $72 \mathrm{~h}$ and limited $\beta$-amyrin production, while it was high from 72 to $144 \mathrm{~h}$ and led to high $\beta$-amyrin productivity. Another possibility was that 2,3-oxidosqualene supply was insufficient for the first $72 \mathrm{~h}$, while it was enough from 72 to $144 \mathrm{~h}$ and led to high $\beta$-amyrin productivity.

$\beta$-amyrin and dammarenediol-II still accumulated in strain GY-1, suggesting that oleanolic acid synthase, protopanaxadiol synthase and protopanaxatriol synthase might be rate-limiting enzymes for aglycon production in this strain. These synthases are P450 enzymes. Poor coupling between P450 cytochromes and their reductases can result in the release of reactive oxygen species $^{37}$, and the CPR levels would thus affect cell health ${ }^{7}$. The CPR gene of A. thaliana (AtCPR1) was used in this study; however, more CPRs from other organisms, such as M. truncatula and G. glabra, must be further investigated to identify the best enzyme for aglycon production.

The artemisinic acid synthetic pathway had been constructed in yeast by using cytochrome P450 CYP71AV1, which can catalyse a three-step, sequential oxidation that can convert amorphadiene to artemisinic acid ${ }^{11}$. However, the product titer was relatively low, only $100 \mathrm{mg} / \mathrm{L}$. Recently, two plant dehydrogenases and a second cytochrome were discovered that could provide a more efficient biosynthetic route toward artemisinic acid. Cytochrome $b_{5}$ (CYB5) facilitated the oxidation of amorphadiene to artemisinic alcohol, alcohol dehydrogenase (ADH1) oxidised artemisinic alcohol to artemisinic aldehyde, while artemisinic aldehyde dehydrogenase (ALDH1) oxidised artemisinic aldehyde to artemisinic acid. After reconstituting the entire, heterologous biosynthetic pathway in yeast, the engineered strain produced $25 \mathrm{~g} / \mathrm{L}$ artemisinic acid ${ }^{7}$. Because oleanolic acid synthase also catalyses a three-step sequential oxidation, it was suggested that identification of other cytochromes and dehydrogenases from plants (such as G. glabra, P. ginseng and $M$. truncatula) and reconstituting them into the engineered yeast would improve oleanolic acid production.

In ginseng, aglycons (protopanaxadiol, protopanaxatriol and oleanolic acid) are further converted to ginsenoside compounds by uridine diphosphate glycosyltransferases (UGTs) ${ }^{18,19}$. Although protopanaxadiol, protopanaxatriol and oleanolic acid have diverse pharmacological effects, ginsenosides (such as Rh2 and Rg3) have more applications in pharmaceutical industries. After functional genomic analysis of $P$. ginseng and other plants, candidate UGTs can be introduced into strain GY-1, which can be used as the host strain for the identification of appropriate UGTs for ginsenoside production.

\section{Methods}

Strains and medium. S. cerevisiae BY4742, a derivative of $\mathrm{S} 288 \mathrm{C}^{38}$, was obtained from EUROSCARF and used as the parent strain for all yeast strains. Engineered yeast strains were grown either in SD medium ${ }^{9,39}$ lacking leucine, uracil, tryptophan and 
histidine, where appropriate, or in YPD medium ${ }^{39}$. Escherichia coli Trans-10 (TransGen Biotech, Beijing, China) was used for transformation and plasmid DNA extraction. Strains were cultivated at $37^{\circ} \mathrm{C}$ in $\mathrm{LB}$ medium with $100 \mathrm{mg} / \mathrm{L}$ ampicillin.

Plasmid construction. Panax ginseng cells were induced with $0.1 \mathrm{mg} / \mathrm{L}$ MeJA for $24 \mathrm{~h}$. Total RNA was isolated by the Trizol method ${ }^{40}$, and cDNA was synthesised using the PrimeScript 1st Strand cDNA Synthesis Kit (Takara, Dalian, China). The $P$. ginseng $\beta$-amyrin synthase ( $P g b A S)$ were PCR-amplified from the cDNA of $P$. ginseng (using primer sets SexA1-PgPNY/PgPNY-Asc1). The amplified DNA fragments were cloned into pEASY-Blunt, resulting in p-PgbAS.

The Glycyrrhiza glabra $\beta$-amyrin synthase gene (GgbAS) (GenBank: AB037203), P. ginseng protopanaxatriol synthase gene (PgPPTS) (GenBank: JX036031) and

Medicago truncatula oleanolic acid synthase gene (MtOAS) (GenBank:FN995113), together with the SexA1 and Asc1 restriction sites at their 5' - and 3' - ends, were synthesised by GenScript (Nanjing, China) with codon optimisation for improved expression in S. cerevisiae. The synthesised DNA fragments were cloned into pUC57, resulting in p-GgbAS, p-SynPgPPTS and p-MtOAS, respectively.

The plasmids of $\mathrm{p}-\Delta \operatorname{Trp} 1, \mathrm{p} \delta$-tHMG1, pM13-LYS2, pM3-ERG9, pM11-ERG1, pM11-AtCPR1, pM2-PgbAS, pM2-GgbAS, pM3-MtOAS, pM3-synPgPPDS, pM14PgDDS, pM8-synPgPPTS, prDNA-LEU, pTrp-HIS and pHis-TRP are described in the supplementary data.

All the plasmids are summarised in Table S1, and the primers used during plasmid construction are summarised in Table S2.

Strain construction. Transformation of $S$. cerevisiae strains was performed by the standard lithium acetate method ${ }^{39}$. Strain BY4742-TRP was constructed by deleting the TRP1 gene of strain BY4742, thus leading to tryptophan auxotrophy and was accomplished as follows. The homologous recombination region of the partial TRP1 gene with the Loxp-HIS3-Loxp cassette was amplified from $\mathrm{p}-\Delta \operatorname{Trp} 1$ using primer sets ZD-TRP1-int-up(400)/ZD-TRP1-int-down(450). The amplified DNA fragment was then transformed into strain BY4742 followed by selection on SD-HIS plates. Strains were verified by PCR analysis, and the correct colony was transformed with plasmid pSH47. The transformant was cultivated at $30^{\circ} \mathrm{C}$ in SD-HIS-URA medium with $2 \%$ galactose as its sole carbon source for $24 \mathrm{~h}$ followed by selection on YPD medium with $1 \mathrm{~g} / \mathrm{L} 5$-FOA as a selection for the loss of plasmid pSH47, resulting in strain BY4742-TRP. This strain could not grow on SD-HIS and SD-TRP plates.

Strain BY-T1 was constructed by integrating the tHMG1 and LYS2 genes into the $\delta D N A$ sites of BY4742-TRP using the DNA assembler method as described previously ${ }^{41,42}$. Three DNA fragments were amplified from $\mathrm{p} \delta$-tHMG1 (using primer sets $1-\mathrm{M}-\mathrm{HisG}-\mathrm{PGK} 1-\mathrm{F} / 1-\mathrm{M}-\mathrm{ADH}-\mathrm{TEF} 1-\mathrm{R}$ and $3-\mathrm{M}-\mathrm{CYC} 1 \mathrm{t}-\delta 2-\mathrm{F} / 3-\delta 2-\mathrm{R})$ and $\mathrm{pM} 13-$ LYS2 (using primer set 2-M-ADHt-TEF1-F/2-M-CYC1t- $\delta 2-\mathrm{R}$ ), and another DNA fragment was obtained by $B a m H I$ and XhoI digestion of plasmid $\mathrm{p} \delta$-UB. These four DNA fragments were transformed into strain BY4742-TRP followed by selection on SD-URA plates. All strains were verified by PCR analysis, and twelve colonies were screened by GC-MS for the selection of the best squalene-producing transformant.

Strain BY- $\beta A-P$ was constructed by integrating the ERG9, ERG1 and PgbAS genes into the $r D N A$ sites of strain BY-T1. Five DNA fragments were amplified from pM2PgbAS (using primer set 1-M-pEASY-PGK1-F/3G-1-M-ADHt-TDH3-R), pM3ERG9 (using primer set 3G-2-M-TPI1t-TEF1-F/M-CYC1t-pEASY-R), pM11-ERG1 (using primer set 3G-3-M-ADHt -TDH3-F/3G-3-M-TPI1t-TEF1-R) and prDNALEU (using primer sets X1-M-pEASY-r-t-F/X1-r-t-R-rDNA and X2-r-t-F-rDNA/ $\mathrm{X} 2-\mathrm{M}-\mathrm{pEASY}-\mathrm{r}-\mathrm{t}-\mathrm{R})$. These fragments were then transformed into strain BY-T1 followed by selection on SD-URA-LEU plates. All strains were verified by PCR analysis, and twelve colonies were screened by GC/MS for the selection of the bestproducing transformant.

BY- $\beta$ A-G was constructed by integrating the ERG9, ERG1 and GgbAS genes into the $r D N A$ sites of strain BY-T1 to construct strain BY- $\beta$ A-P.

BY- $\beta A-C K$ was constructed by integrating the LYS2 and GgbAS genes into the rDNA sites of strain BY4742-TRP. Four DNA fragments were amplified from pM2GgbAS (using primer set 1-M-pEASY-PGK1-F/1-M-ADHt-TEF1-R), pM13-LYS2 (using primer set 2-M-ADHt-TEF1 -F/M-CYC1t-pEASY-R) and prDNA-LEU (using primer sets X1-M-pEASY-r-t-F/X1-r-t-R-rDNA and X2-r-t-F-rDNA/X2-MpEASY-r-t-R) and were then transformed into strain BY4742-TRP followed by selection on SD-LEU plates. All strains were verified by PCR analysis, and 12 colonies were screened by GC/MS for the selection of the best-producing transformant.

Strain BY-OA was constructed by integrating the GgbAS, MtOAS and AtCPR1 genes into the Trp 1 site of BY- $\beta A-G$. Five DNA fragments were amplified from $\mathrm{pM} 2$ GgbAS (using primer set 1-M-pEASY-PGK1-F/3G-1-M-ADHt-TDH3-R), pM3MtOAS (using primer set 3G-2-M-TPI1t-TEF1-F/M-CYC1t-pEASY-R), pM11AtCPR1 (using primer set 3G-3-M-ADHt-TDH3-F/3G-3-M-TPI1t-TEF1-R) and pTrp-HIS (using primer sets X1-M-pEASY-r-t-F/ZD-TRP1interg.-1 and ZDTRP1interg.-2/X2-M-pEASY-r-t-R) and were then transformed into strain BY- $\beta A-G$ followed by selection on SD-URA-LEU-HIS plates. All strains were verified by PCR analysis, and 12 colonies were screened by GC/MS and HPLC for selection of the bestproducing transformant.

Strain GY-1 was constructed by integrating the PgDDS, SynPgPPDS, SynPPTS and AtCPR1 genes into the His3 site of BY-OA. Six DNA fragments were amplified from pM14-PgDDS (using primer set 1-M-pEASY-PGK1-F/4G-1-M-ADHt-FBA1-R), pM8-SynPgPPTS (using primer set 4G-4-M-ADHt-FBA1-F/4G-4-M-TDH2tTDH3-R), pM11-AtCPR1 (using primer set 4G-3-M-TDH2t-TDH3-F/3G-3-MTPI1t-TEF1-R), pM3-SynPgPPDS (using primer set 3G-2-M-TPI1t-TEF1-F/MCYC1t-pEASY-R) and pHis-TRP (using primer sets X1-M-pEASY-r-t-F/ZD-His3 interg.-1 and ZD-His3 interg.-2/X2-M-pEASY-r-t-R) and were transformed into strain BY-OA followed by selection on SD-URA-LEU-HIS-TRP plates. All strains were verified by PCR analysis, and 12 colonies were screened by GC/MS and HPLC for the selection of the best-producing transformant.

Primers used in the DNA assembly are summarised in Table S3.

Yeast cultivation. YPD medium was used for cultivating strains BY4742, BY-T1, BY$\beta A-P, B Y-\beta A-G, B Y-\beta A-C K, B Y-O A$ and GY-1. All strains were first inoculated into $15 \mathrm{ml}$ culture tubes containing $2 \mathrm{ml}$ medium and grown at $30^{\circ} \mathrm{C}$ and $250 \mathrm{rpm}$ to an $\mathrm{OD}_{600}$ of approximately 1.0. Flasks $(250 \mathrm{ml})$ containing $100 \mathrm{ml}$ medium were then inoculated to an $\mathrm{OD}_{600} 0.05$ with the seed cultures. Strains were grown at $30^{\circ} \mathrm{C}$ and $250 \mathrm{rpm}$ for 7 days, and all optical densities at $600 \mathrm{~nm}\left(\mathrm{OD}_{600}\right)$ were measured using a Shimadzu UV-2550 spectrophotometer.

Chemical analysis. Cells were collected from fermentation culture via centrifugation. The mixed-solution $(600 \mu \mathrm{l}$; acetone: methanol=1:1) was added to the tube and crushed by a BeadBeater (BioSpec, USA) 3 times. The samples were then centrifuged at $10,000 \times g$ for $1 \mathrm{~min}$, and $1 \mu \mathrm{l}$ of supernatant was analysed by GC/MS using an Agilent technologies 5975C insert xl MSD with triple-Axis Detector equipped with a HP- $5 \mathrm{~ms}(30 \mathrm{~m} \times 0.25 \mathrm{~mm} \times 0.5 \mu \mathrm{m}) \mathrm{GC}$ column. Compound separation was achieved with an injector temperature of $300^{\circ} \mathrm{C}$ and a $30 \mathrm{~min}$ temperature gradient program for GC-separation starting at $80^{\circ} \mathrm{C}$ for $1 \mathrm{~min}$ followed by heating the column to $300^{\circ} \mathrm{C}$ at $20^{\circ} \mathrm{C} \mathrm{min}$ min $^{-1}$ and a final constant hold at $300^{\circ} \mathrm{C}$ for $15 \mathrm{~min}$. Mass detection was achieved with electric ionisation using SIM-scan mode with diagnostic ions monitored as followed: $\mathrm{m} / \mathrm{z} 69, \mathrm{~m} / \mathrm{z} 218, \mathrm{~m} / \mathrm{z} 363, \mathrm{~m} / \mathrm{z} 411$ and $\mathrm{m} / \mathrm{z} 437$. A crystallised $\beta$-amyrin sample was used as the standard for quantification (purchased from BioBioPha, China), and squalene, lanosterol and ergosterol standards (purchased from Sigma Aldrich) were also used for quantification.

For the determination of oleanolic acid, acetone and methanol $(1: 1)$ extracts $(20 \mu \mathrm{l})$ were analysed by LC/MS using an Agilent 1200 HPLC system coupled to a Bruker-micrOTOF-II with an electrospray ionisation (ESI) interface. Data acquisition and processing were performed with the MicrOTOF control version 3.0/Data Analysis Version 4.0 software. For chromatographic separation, a Waters Symmetry $\mathrm{C} 18^{\circledR}$ column $(250 \mathrm{~mm} \times 4.6 \mathrm{~mm}, 5 \mu \mathrm{m})$ was used. The mobile phase consisted of $0.1 \%$ ammonium acetate in water (A) and acetonitrile (B), and a program of $\mathrm{A}: \mathrm{B}=15: 85$ for 30 minutes was used. The solvent flow rate was $1.0 \mathrm{~mL} / \mathrm{min}$ and the column temperature was set at $30^{\circ} \mathrm{C}$. Optimised MS operating conditions were as follows: all spectra were obtained in the negative ion mode over an $\mathrm{m} / \mathrm{z}$ range of $100-$ 1200; dry gas flow, $6.0 \mathrm{~L} / \mathrm{min}$; dry temperature, $180^{\circ} \mathrm{C}$; nebuliser pressure, 1 bar; and probe voltage, $-4.5 \mathrm{kV}$. Oleanolic acid was purchased from Sigma Aldrich and was used as the standard for analysis.

For the determination of protopanaxadiol and protopanaxatriol, acetone and methanol $(1: 1)$ extracts $(20 \mu \mathrm{l})$ were analysed by LC/MS using an Agilent 1200 HPLC system coupled to a Bruker-micrOTOF-II with an electrospray ionisation (ESI) interface. Data acquisition and processing were performed with the MicrOTOF control version 3.0/Data Analysis Version 4.0 software. For chromatographic separation, a Waters Symmetry C18 ${ }^{\circledR}$ column $(250 \mathrm{~mm} \times 4.6 \mathrm{~mm}, 5 \mu \mathrm{m})$ was used. The mobile phase consisted of $0.1 \%$ formic acid and $10 \%$ methanol in water $(\mathrm{A})$ and acetonitrile (B), and a program of $\mathrm{A}: \mathrm{B}=15: 85$ for 30 minutes was used. The solvent flow rate was $1.0 \mathrm{~mL} / \mathrm{min}$, and the column temperature was set at $30^{\circ} \mathrm{C}$. The optimised MS operating conditions were as follows: all spectra were obtained in the positive ion mode over an $\mathrm{m} / \mathrm{z}$ range of 100-1200; dry gas flow, $6.0 \mathrm{~L} / \mathrm{min}$; dry temperature, $180^{\circ} \mathrm{C}$; nebuliser pressure, 1 bar; and probe voltage $+4.5 \mathrm{kV}$. Protopanaxadiol and protopanaxatriol was purchased from Sigma Aldrich and was used as the standard for analysis.

For quantitative analysis of protopanaxadiol, protopanaxatriol and oleanolic acid, acetone and methanol $(1: 1)$ extracts $(20 \mu \mathrm{l})$ were injected into an Agilent 1200 HPLC apparatus with UV detection at $203 \mathrm{~nm}$. For chromatographic separation, a Waters Symmetry C18 ${ }^{\circledR}$ column $(250 \mathrm{~mm} \times 4.6 \mathrm{~mm}, 5 \mu \mathrm{m})$ was used. The mobile phase consisted of $0.1 \%$ Formic acid and $10 \%$ methanol in water (A) and acetonitrile (B), and a program of $\mathrm{A}: \mathrm{B}=15: 85$ for 30 minutes was used. The solvent flow rate was $1.0 \mathrm{~mL} / \mathrm{min}$, and the column was held at $30^{\circ} \mathrm{C}$ during the separation. Protopanaxadiol, Protopanaxatriol and oleanolic acid was purchased from Sigma Aldrich and was used for quantification.

1. Qi, L. W., Wang, C. Z. \& Yuan, C. S. Ginsenosides from American ginseng: chemical and pharmacological diversity. Phytochemistry 72, 689-699 (2011).

2. Radad, K., Moldzio, R. \& Rausch, W. D. Ginsenosides and their CNS targets. CNS Neurosci Ther 17, 761-768 (2011).

3. Han, J. Y., Kim, H. J., Kwon, Y. S. \& Choi, Y. E. The Cyt P450 enzyme CYP716A47 catalyzes the formation of protopanaxadiol from dammarenediol-II during ginsenoside biosynthesis in Panax ginseng. Plant Cell Physiol 52, 2062-2073 (2011).

4. Paek, K. Y., Murthy, H. N., Hahn, E. J. \& Zhong, J. J. Large scale culture of ginseng adventitious roots for production of ginsenosides. Adv Biochem Eng Biotechnol 113, 151-176 (2009).

5. Ajikumar, P. K. et al. Terpenoids: opportunities for biosynthesis of natural product drugs using engineered microorganisms. Mol Pharm 5, 167-190 (2008).

6. Chang, M. C. \& Keasling, J. D. Production of isoprenoid pharmaceuticals by engineered microbes. Nat Chem Biol 2, 674-681 (2006). 
7. Paddon, C. J. et al. High-level semi-synthetic production of the potent antimalarial artemisinin. Nature 496, 528-532 (2013).

8. Dejong, J. M. et al. Genetic engineering of taxol biosynthetic genes in Saccharomyces cerevisiae. Biotechnol Bioeng 93, 212-224 (2006).

9. Dai, Z., Liu, Y., Huang, L. \& Zhang, X. Production of miltiradiene by metabolically engineered Saccharomyces cerevisiae. Biotechnol Bioeng 109, 2845-2853 (2012).

10. Engels, B., Dahm, P. \& Jennewein, S. Metabolic engineering of taxadiene biosynthesis in yeast as a first step towards Taxol (Paclitaxel) production. Metab Eng 10, 201-206 (2008).

11. Ro, D. K. et al. Production of the antimalarial drug precursor artemisinic acid in engineered yeast. Nature 440, 940-943 (2006)

12. Westfall, P. J. et al. Production of amorphadiene in yeast, and its conversion to dihydroartemisinic acid, precursor to the antimalarial agent artemisinin. P Natl Acad Sci USA 109, E111-118 (2012).

13. Carelli, M. et al. Medicago truncatula CYP716A12 is a multifunctional oxidase involved in the biosynthesis of hemolytic saponins. Plant Cell 23, 3070-3081 (2011).

14. Fukushima, E. O. et al. CYP716A subfamily members are multifunctional oxidases in triterpenoid biosynthesis. Plant cell physiology 52, 2050-2061 (2011).

15. Huang, L. et al. Molecular characterization of the pentacyclic triterpenoid biosynthetic pathway in Catharanthus roseus. Planta 236, 1571-1581 (2012)

16. Han, J. Y. et al. Cytochrome P450 CYP716A53v2 catalyzes the formation of protopanaxatriol from protopanaxadiol during ginsenoside biosynthesis in Panax ginseng. Plant Cell Physiol 53, 1535-1545 (2012).

17. Tansakul, P., Shibuya, M., Kushiro, T. \& Ebizuka, Y. Dammarenediol-II synthase, the first dedicated enzyme for ginsenoside biosynthesis, in Panax ginseng. FEBS Lett 580, 5143-5149 (2006).

18. Augustin, J. M., Kuzina, V., Andersen, S. B. \& Bak, S. Molecular activities, biosynthesis and evolution of triterpenoid saponins. Phytochemistry 72, 435-457 (2011).

19. Chen, S. et al. 454 EST analysis detects genes putatively involved in ginsenoside biosynthesis in Panax ginseng. Plant Cell Rep 30, 1593-1601 (2011).

20. Kennedy, M. A., Barbuch, R. \& Bard, M. Transcriptional regulation of the squalene synthase gene (ERG9) in the yeast Saccharomyces cerevisiae. Biochim Biophys Acta 1445, 110-122 (1999).

21. Leber, R. et al. A novel sequence element is involved in the transcriptional regulation of expression of the ERG1 (squalene epoxidase) gene in Saccharomyces cerevisiae. European journal of biochemistry/FEBS 268, 914-924 (2001).

22. Ma, S. M. et al. Optimization of a heterologous mevalonate pathway through the use of variant HMG-CoA reductases. Metab Eng 13, 588-597 (2011).

23. Seki, H. et al. Triterpene functional genomics in licorice for identification of CYP72A154 involved in the biosynthesis of glycyrrhizin. The Plant cell 23 4112-4123 (2011)

24. Urban, P., Mignotte, C., Kazmaier, M., Delorme, F. \& Pompon, D. Cloning, yeast expression, and characterization of the coupling of two distantly related Arabidopsis thaliana NADPH-cytochrome P450 reductases with P450 CYP73A5. J BIOL CHEM 272, 19176-19186 (1997).

25. Veen, M., Stahl, U. \& Lang, C. Combined overexpression of genes of the ergosterol biosynthetic pathway leads to accumulation of sterols in Saccharomyces cerevisiae. FEMS Yeast Res 4, 87-95 (2003).

26. Albertsen, L. et al. Diversion of flux toward sesquiterpene production in Saccharomyces cerevisiae by fusion of host and heterologous enzymes. Appl Environ Microbiol 77, 1033-1040 (2011).

27. Asadollahi, M. A., Maury, J., Schalk, M., Clark, A. \& Nielsen, J. Enhancement of farnesyl diphosphate pool as direct precursor of sesquiterpenes through metabolic engineering of the mevalonate pathway in Saccharomyces cerevisiae. Biotechnol Bioeng 106, 86-96 (2010).

28. Zhou, Y. J. et al. Modular pathway engineering of diterpenoid synthases and the mevalonic acid pathway for miltiradiene production. J Am Chem Soc 134, 3234-3241 (2012).

29. Kirby, J., Romanini, D. W., Paradise, E. M. \& Keasling, J. D. Engineering triterpene production in Saccharomyces cerevisiae beta-amyrin synthase from Artemisia annua. FEBS J 275, 1852-1859 (2008).

30. Madsen, K. M. et al. Linking genotype and phenotype of Saccharomyces cerevisiae strains reveals metabolic engineering targets and leads to triterpene hyperproducers. PLoS One 6, el4763 (2011).
31. Verwaal, R. et al. High-level production of beta-carotene in Saccharomyces cerevisiae by successive transformation with carotenogenic genes from Xanthophyllomyces dendrorhous. Appl Environ Microbiol 73, 4342-4350 (2007)

32. Leber, R. et al. A novel sequence element is involved in the transcriptional regulation of expression of the ERG1 (squalene epoxidase) gene in Saccharomyces cerevisiae. Eur J Biochem 268, 914-924 (2001)

33. Kim, Y. S. et al. Gene regulation patterns in triterpene biosynthetic pathway driven by overexpression of squalene synthase and methyl jasmonate elicitation in Bupleurum falcatum. Planta 233, 343-355 (2011).

34. Lee, M. H. et al. Enhanced triterpene and phytosterol biosynthesis in Panax ginseng overexpressing squalene synthase gene. Plant cell physiology 45, 976-984 (2004).

35. Seo, J. W. et al. Overexpression of squalene synthase in Eleutherococcus senticosus increases phytosterol and triterpene accumulation. Phytochemistry 66, 869-877 (2005).

36. Seki, H. et al. Licorice beta-amyrin 11-oxidase, a cytochrome $\mathrm{P} 450$ with a key role in the biosynthesis of the triterpene sweetener glycyrrhizin. Proc Natl Acad Sci USA 105, 14204-14209 (2008).

37. Zangar, R. C., Davydov, D. R. \& Verma, S. Mechanisms that regulate production of reactive oxygen species by cytochrome P450. Toxicol Appl Pharmacol 199, 316-331 (2004).

38. Brachmann, C. B. et al. Designer deletion strains derived from Saccharomyces cerevisiae S288C: a useful set of strains and plasmids for PCR-mediated gene disruption and other applications. Yeast 14, 115-132 (1998).

39. Burke, D., Dawson, D. \& Stearns, T. Methods in yeast genetics: a cold spring harbor laboratory course manual (Cold Spring Harbor Laboratory Press, New York, 2000).

40. Dai, Z., Cui, G., Zhou, S. F., Zhang, X. \& Huang, L. Cloning and characterization of a novel 3-hydroxy-3-methylglutaryl coenzyme A reductase gene from Salvia miltiorrhiza involved in diterpenoid tanshinone accumulation. J Plant Physiol 168, 148-157 (2011).

41. Shao, Z. \& Zhao, H. DNA assembler, an in vivo genetic method for rapid construction of biochemical pathways. Nucleic Acids Res 37, e16 (2009).

42. Dai, Z. et al. Metabolic engineering of Saccharomyces cerevisiae for production of ginsenosides. Metab Eng 20, 146-156 (2013).

\section{Acknowledgments}

This research was supported by grants from the National Basic Research Program of China (2011CBA00800), National High Technology Research and Development Program (2012AA02A704) and the National Natural Science Foundation of China (81202864, 81130070 and 81072989 ). Xueli Zhang was supported by the Hundred Talent Program of the Chinese Academy of Sciences. We thank Dr. N.A. Da Silva (University of California) for providing the plasmid $\mathrm{p} \delta$-UB, and Dr. Qinhong Wang (Tianjin Institute of Industrial Biotechnology, Chinese Academy of Sciences) for providing the yeast vectors pRS313, pRS314.

\section{Author contributions}

Z.D., L.H., T.L. and X.Z. designed the experiments; Z.D., Y.L., B.W., M.S., D.W. and X.N.Z. performed the experiments; Y.L., Z.D. and X.Z. analysed the data; Z.D. and X.Z. wrote the paper; and all authors reviewed the manuscript.

\section{Additional information}

Supplementary information accompanies this paper at http://www.nature.com/ scientificreports

Competing financial interests: This work has been included in a patent application by the Tianjin Institute of Industrial Biotechnology.

How to cite this article: Dai, Z.B. et al. Producing aglycons of ginsenosides in bakers' yeast. Sci. Rep. 4, 3698; DOI:10.1038/srep03698 (2014)

(2) This work is licensed under a Creative Commons AttributionBY NC SA NonCommercial-ShareAlike 3.0 Unported license. To view a copy of this license, visit http://creativecommons.org/licenses/by-nc-sa/3.0 